\title{
PAPER
}

\section{Do cognitive patterns of brain magnetic activity correlate with hippocampal atrophy in Alzheimer's disease?}

\author{
F Maestú, J Arrazola, A Fernández, P G Simos, C Amo, P Gil-Gregorio, S Fernandez, \\ A Papanicolaou, T Ortiz
}

See Editorial Commentary

p 147

J Neurol Neurosurg Psychiatry 2003;74:208-212

See end of article for

authors' affiliations

.....................

Correspondence to:

Dr Tomas Ortiz, Centro de Magnetoencefalografía Dr

Pérez Modrego, Pabellón

No 8 Facultad de

Medicina, Universidad

Complutense de Madrid

28040, Madrid, Spain;

cmeg@eucmos.sim.ucm.es

Received 4 April 2002

In revised form 27 August

2002

Accepted 4 September 2002

Background: Many reports support the clinical validity of volumetric MRI measurements in Alzheimer's disease.

Objective: To integrate functional brain imaging data derived from magnetoencephalography (MEG) and volumetric data in patients with Alzheimer's disease and in age matched controls.

Methods: MEG data were obtained in the context of a probe-letter memory task. Volumetric measurements were obtained for lateral and mesial temporal lobe regions.

Results: As expected, Alzheimer's disease patients showed greater hippocampal atrophy than controls bilaterally. MEG derived indices of the degree of activation in left parietal and temporal lobe areas, occurring after $400 \mathrm{~ms}$ from stimulus onset, correlated significantly with the relative volume of lateral and mesial temporal regions. In addition, the size of the right hippocampus accounted for a significant portion of the variance in cognitive scores independently of brain activity measures.

Conclusions: These data support the view that there is a relation between hippocampal atrophy and the degree of neurophysiological activity in the left temporal lobe.

P rogressive cortical atrophy, especially in the temporal lobe, is one of the most important macroscopic findings in patients with Alzheimer's disease. Magnetic resonance imaging (MRI) has sufficient spatial resolution to allow detailed assessment of cortical grey matter loss in vivo ${ }^{1}$ and serves as a valuable clinical tool in certain neurological conditions such as epilepsy. ${ }^{2}$ Quantitative measures of cortical atrophy, using MRI volumetry, can distinguish between Alzheimer's disease and elderly control patients ${ }^{3}$ with a high degree of sensitivity and specificity. There is evidence supporting the concurrent validity of these measures as indices of regional neuronal loss, and their predictive validity as an early sign of the disease. ${ }^{5}$ Moreover, there is close agreement between volumetric and cognitive measures in differentiating patients from normal controls. ${ }^{6}$ The degree of hippocampal ${ }^{7}$ or parahippocampal atrophy ${ }^{8}$ has been related to the severity of memory impairment. While many studies have focused on the hippocampal formation and the adjacent entorhinal cortex, ${ }^{9}$ others found evidence implicating neocortical areas, including the lateral surface of the temporal lobe and the parietal operculum in the left hemisphere, with relative sparing of sensorimotor and visual areas. ${ }^{1}$ These reports are in agreement with metabolic, ${ }^{10}$ blood flow, ${ }^{11}$ and slow wave magnetoencephalographic findings. ${ }^{12}$

Magnetoencephalography (MEG) is a non-invasive functional brain imaging technique that can show, in real time, spatiotemporal patterns of regional neurophysiological activity. Recent MEG studies of Alzheimer's disease have produced very promising results that support the potential clinical value of the technique. ${ }^{12}{ }^{13}$ Aberrant patterns of brain activity in patients with Alzheimer's disease have been found in records of ongoing magnetic measurements, ${ }^{14}$ as well as in the context of event related activation protocols. ${ }^{15}$ In the latter study, spatiotemporal brain activation profiles obtained from patients with Alzheimer's disease during performance of a working memory task showed reduced activation in temporal and parietal areas in comparison with elderly controls. The degree of activity in this region was a significant predictor of scores in cognitive and daily function scales. Consistent with the notion that neuropsychological deficits in higher order association areas underlie the most severe cognitive impairments in Alzheimer's disease, only activity occurring in the late phases of stimulus processing (that is, after $400 \mathrm{~ms}$ poststimulus) was significantly related to indices of cognitive function.

This study extends these findings by examining the concurrent validity of both functional and anatomical measures obtained from the same patients and elderly control subjects. In this way we can assess the complementarity both of MEG derived measures of task related regional cerebral activation and of indices of cortical atrophy as correlates of cognitive function in patients diagnosed with Alzheimer's disease. If the two sets of data provide independent but related information on the status of the brain, they may, when combined, serve as significantly better indicators of the severity of the disease and as more reliable predictors of the course of the illness.

\section{METHODS}

\section{Subjects}

Eight patients (mean (SD) age, 76.8 (1.01) years) from the Hospital Universitario San Carlos de Madrid Geriatric unit fulfilling the NINCDS-ADRDA criteria of probable Alzheimer's

Abbreviations: CAMCOG, Cambridge cognitive examination; $\mathrm{CVr}$ relative cerebral volume; $E C D$, equivalent current dipole; $E R F$, event related magnetic flux; FA, flip angle; FAST, functional assessment scale; $\mathrm{LHV}$, left hippocampal volume; LHVr, proportion of the total cranial volume represented by the left hippocampus; LTLS, left lateral surface of the temporal lobe; LTLSr, proportion of the total cranial volume represented by the left temporal lobe; MEG, magnetoencephalography; MMSE, mini-mental state examination; NINCDS-ADRDA, National Institute of Neurological and Communicative Disorders and Stroke-Alzheimer's Disease and Related Disorders Association; RHV, right left hippocampal volume; $\mathrm{RHVr}$, proportion of the total cranial volume represented by the right hippocampus; RTLS, right lateral surface of the temporal lobe; RTLSr, proportion of the total cranial volume represented by the right temporal lobe; $\mathrm{TCrV}$, total cranial volume; TCV, total cerebral volume; TE, time of excitation; TR, time of repetition 
Table 1 Group scores on the mini-mental state examination (Spanish version, maximum score $=35$ ), CAMCOG, and FAST

\begin{tabular}{lll}
\hline Test & Group & Mean (SD) \\
\hline FAST & Alzheimer & $3.5(0.5)$ \\
& Control & $1.4(0.6)^{*}$ \\
MMSE & Alzheimer & $20.3(2.2)$ \\
& Control & $33.7(1.4)^{*}$ \\
CAMCOG & Alzheimer & $56.2(6.09)$ \\
& Control & $87.8(4.2)^{*}$
\end{tabular}

${ }^{*} p<0.01$ (tests of group differences). CAMCOG, Cambridge cognitive examination; FAST, functional assessment scale; MMSE mini-mental state examination (Spanish version).

disease, and eight age matched cognitively intact control subjects (age 75.62 (1.87) years) participated in the study. MEG data from the same patients have been reported in a previous communication. ${ }^{15}$ In addition to the standard battery of neuropsychological tests administered in the clinic, patients and controls received two specialised clinical tests of cognitive function, the Cambridge cognitive examination (CAMCOG) and the mini-mental state examination (MMSE). The Spanish version of the $\mathrm{MMSE}^{16}$ has a maximum score of 35 rather than 30 as in the English version. Information on the participants' level of function in every day adaptive activities was obtained using the functional assessment scale (FAST). Table 1 presents the score in each of those test for both groups.

All subjects signed a consent form before participating in the study.

\section{Stimuli and task}

A detailed description of the task can be found in a report by Maestú et $a l,{ }^{15}$ and will be summarised here briefly. Scans were obtained in the context of a letter-probe task in which five letters were presented simultaneously, followed by a series of single letters presented one at a time. The subjects were instructed to hold the items from the initial set in memory and to respond by raising their right index finger upon detecting a letter from that set during the subsequent serial presentation. In all, 250 letter stimuli were presented, $50 \%$ of which were targets (that is, items included in the immediately preceding set) and 50\% were distractors (letters not included in the immediately preceding set).

\section{MEG data collection and analysis}

The MEG signal was measured using a 148 channel whole head magnetometer (Magnes ${ }^{\circledast} 2500 \mathrm{WH}, 4-\mathrm{D}$ Neuroimaging, San Diego, California, USA) in a magnetically shielded room. A minimum of 90 epochs (one second MEG data segments) was used to calculate the average event related magnetic flux waveform (ERF) in each condition. Although different investigators have proposed a variety of source modelling approaches, we relied on the single equivalent current dipole (ECD) source model which is part of the 4D Neuroimaging software.

The intracranial generators (that is, activity sources) of the magnetic signals at successive $4 \mathrm{~ms}$ intervals during the course of the ERF waveform were modelled using a finite version of the non-linear Levenberg-Marquardt algorithm. ${ }^{17}$ Alternative algorithms hold much promise as tools for magnetic source localisation; however, they have not yet been validated against invasive localisation procedures. Currently, this model is part of the standard analysis protocol in essentially all clinical applications of MEG. Moreover, in a variety of both clinical and research applications, a single activity source is sufficient to account for $90-95 \%$ of the variance in the ERF data recorded at a given time point following stimulus onset. Most importantly, the localisation accuracy of the single ECD model for activity sources reflecting cognitive neurophysiological operations is excellent when compared with the results of invasive electrical stimulation mapping studies. ${ }^{18}{ }^{19}$ The algorithm used in this study searched for the activity source that was most likely to have produced the observed magnetic field distribution at a given time. The location of activity sources was computed with reference to a Cartesian coordinate system, defined by a set of three anatomical landmarks (fiduciary points): the right and left external meatus and the nasion. The position of the magnetometers relative to the subject's head was determined precisely using five coils, three of which were attached to the fiduciary points and two on the forehead. The coils were activated briefly at the beginning and again at the end of the recording session, and their precise location in three dimensional space was determined using a localisation algorithm built into the system. During the recording session a fibreoptic motion detector was used to ensure that the subject's head did not change position relative to the sensor.

Activity source solutions were considered as satisfactory upon meeting the following criteria: (1) correlation and goodness of fit $\geqslant 0.90$ between the observed and the best predicted magnetic field distribution; (2) a 95\% confidence volume of less than $10 \mathrm{~cm}^{3}$.

In order to identify the anatomical regions where the activity sources were localised, activity source coordinates were overlaid onto $\mathrm{Tl}$ weighted magnetic resonance images using the STAR software which is part of the 4D Neuroimaging software. Precise coregistration of the MEG coordinate system onto the MRI was achieved by aligning the MEG fiduciary points with high contrast cod liver capsules $(3 \mathrm{~mm}$ in diameter) which were fixed to the subject's nasion and inserted in the external meatus before the MRI scan.

The sum of all acceptable sources localised in a particular brain region, starting at stimulus onset and ending one second later, served as a metric of the degree of stimulus locked activation of that area. The validity of this measure as an index of regional activation has been established in several studies involving neurologically intact volunteers and patients. ${ }^{20}{ }^{21}$ Further, in order to extract information regarding the relative timing of group differences, the number of activity sources in each area was summed within each of 10 consecutive $100 \mathrm{~ms}$ time windows. The following variables, representing the total number of activity sources in response to target stimuli, were associated with group ANOVA (analysis of variance) effects in our previous study, ${ }^{15}$ and were considered in the present study: (1) left temporal lobe activity (including activity in the hippocampus) in the 400-500 and 600-700 ms time windows; (2) left parietal activity in the 400-500 and 600-700 ms latency windows.

\section{MRI volumetry}

High resolution three dimensional volume scans were acquired from each subject with a 1.5 Tesla scanner (Signa, General Electric (GE), Milwaukee, Wisconsin, USA). Tl weighted images (TR $14.6 \mathrm{~ms} / \mathrm{TE} 3.1 \mathrm{~ms} / \mathrm{FA} 15^{\circ}$ ) were obtained based on gradient echo (3D-SPGR) sequences with a field of view of $22 \times 16 \mathrm{~cm}, 256 \times 194$ matrix, one excitation, and 1 $\mathrm{mm}$ slice thickness, covering the entire brain and skull in the axial plane. The voxel size was $0.7 \mathrm{~mm}^{3}$. The volumetric measurements were done with the RM tissue volume software developed by GE Medical Systems running on an Advantage Windows 4.0 environment. Regions of interest in each scan were defined using the semi-automatic volume segmentation protocol (3SAVS) which is part of the software. This software allows the user to draw a region of interest in each slice with the mouse, assisted by border attraction and three dimensional automatic paint tools, and measure the volume of the painted region (in $\mathrm{cm}^{3}$ ).

After the measures have been obtained the software produces a histogram of the voxel value for the region of interest. The following regions of interest were defined: total cranial volume (TCrV), total cerebral volume (TCV), right and left hippocampal 


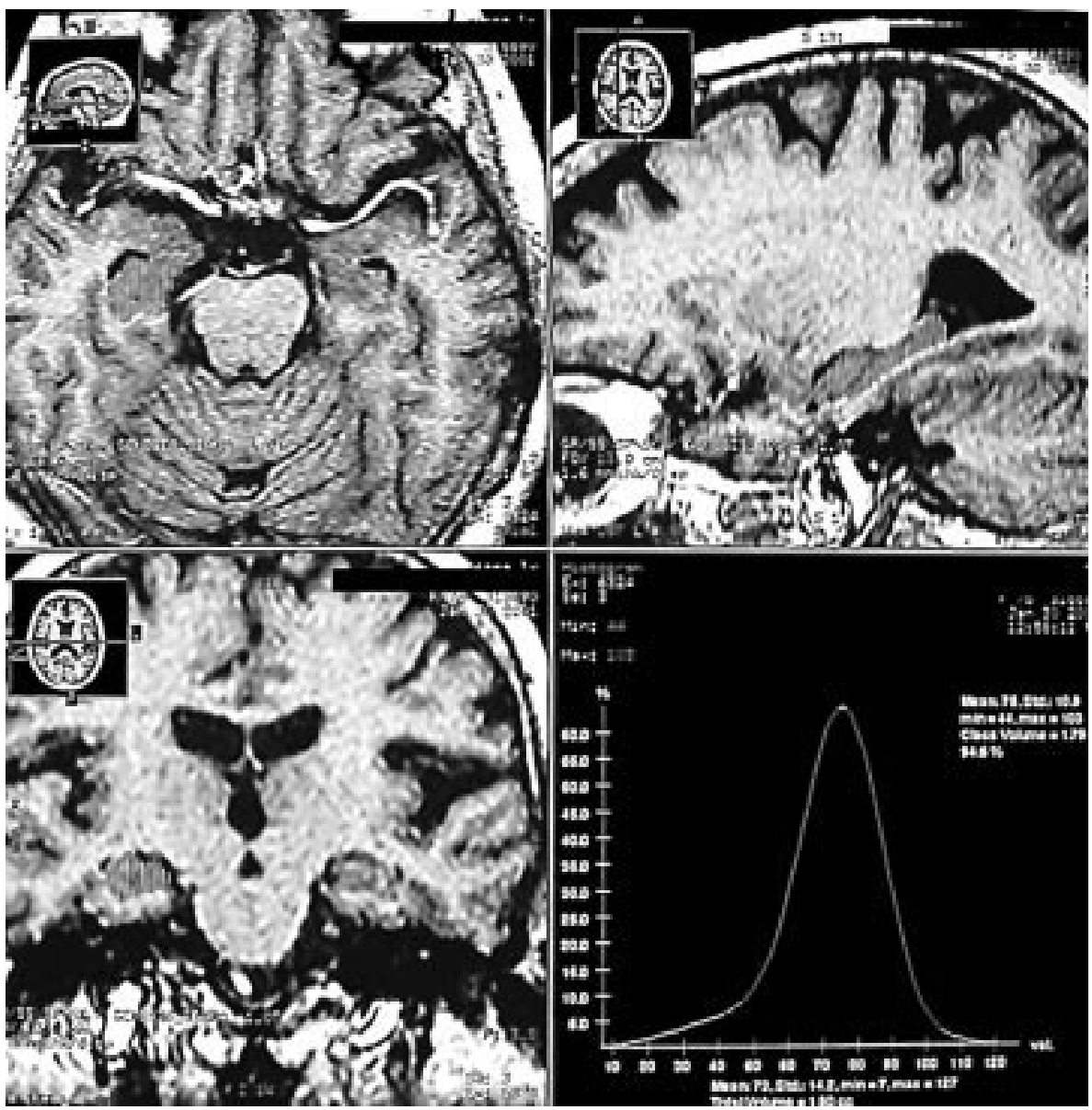

Figure 1 Volumetric MRI data (lower right panel) reflecting the spatial extent of the right hippocampus, in a representative subject, indicated in axial (upper left panel), sagittal (upper right panel), and coronal views (lower left panel).

volume (RHV, LHV), and right and left lateral surface of the temporal lobe (RTLS, LTLS) (fig 1). In order to determine anatomical landmarks on the lateral temporal surface, the Sylvian fissure was traced and the parieto-occipital fissure and the preoccipital notch were identified and joined by a line, following procedures described by Kidron et al. ${ }^{3}$ Then a line was draw from the posterior end of the Sylvian fissure to the line joining the parieto-occipital fissure and preoccipital notch to provide the posterior demarcations of the temporal lobe. After this the ratio of total cerebral to total cranial volume (relative cerebral volume or $\mathrm{CVr}$ ) was calculated according to the formula:

$$
\mathrm{CVr}=\mathrm{TCV} / \mathrm{TCrV} \times 100 .
$$

In a similar manner we computed the proportion of the total cranial volume represented by: the right hippocampus (RHVr), the left hippocampus (LHVr), the right temporal lobe (RTLSr), and the left temporal lobe (LTLSr). This approach was introduced by Whitell et $a^{22}$ in order to control for individual differences in brain size resulting from normal intersubject variation, as well as variation caused by brain pathology. Only relative measures were used in the statistical analyses in order to control for possible group differences in brain size.

To determine the reliability of the MRI measures, we estimated the degree of agreement between the two neuroradiologists who did the volumetric measurements independently without knowledge of the clinical or MEG results. The mean difference in measurements between the two observers for the left hippocampus was $-0.03 \mathrm{~cm}^{3}$ (95\% confidence interval -0.14 to $0.08 \mathrm{~cm}^{3}$ ) and $0.07 \mathrm{~cm}^{3}$ for the right hippocampus $\left(-0.3\right.$ to $\left.0.1 \mathrm{~cm}^{3}\right)$. Similar results were found for the left temporal lobe $\left(-0.1 \mathrm{~cm}^{3}\left(-0.98\right.\right.$ to $\left.\left.0.75 \mathrm{~cm}^{3}\right)\right)$ and for the right temporal lobe $\left(0.17 \mathrm{~cm}^{3}\left(-0.5\right.\right.$ to $\left.0.2 \mathrm{~cm}^{3}\right)$. Interrater reliability coefficients were very high (left hippocampus, $r=0.99$; right hippocampus, $r=0.98$; left temporal lobe, $r=0.94$; right temporal lobe, $r=0.95)$. Corresponding coefficients for the parietal lobes were much lower $(<0.70)$, in part because of the inherent difficulty in defining and visually identifying anatomical borders for this area. Accordingly, parietal volumes were not used in subsequent analyses.

\section{RESULTS}

\section{Volumetric MRI analyses: group effects}

A series of mean comparisons (Student $t$ tests) between groups was performed on the MRI volumetric variables. Given a total number of five comparisons (CVr, RHVr, LHVr, RTLSr, LTLSr), an adjusted $\alpha$ level of $0.05 / 5=0.007$ was used to evaluate each $t$ test by the Bonferroni method. The Alzheimer's disease group had lower volumes, adjusted for total intracranial volume, than the elderly control group in both the left $(\mathrm{t}(14)=5.68, \mathrm{p}<0.0001)$ and the right hippocampus $(t(14)=5.69, p<0.0001)$. The two groups did not differ on the other relative volume measures $(p>0.2)$.

\section{Relations between volumetric MRI and MEG data}

A series of Pearson correlation coefficients was computed between the MEG and the MRI volumetric variables. We focused on the two volumetric variables that reliably differentiated between groups, namely relative left and relative right hippocampal volumes. Three additional variables were examined for contrast: total brain volume (adjusted for head size) and the relative volume of the entire left and the entire right temporal lobes. Two MEG based activity measures were 
Table 2 Correlation coefficients between MEG and MRI variables

\begin{tabular}{lllllll}
\hline \multicolumn{9}{c}{ MRI } & & & \\
\cline { 2 - 6 } & L hippocampus & R hippocampus & Brain & L temporal & R temporal \\
\hline MEG L temporal & $0.70 \ddagger$ & $0.59^{*}$ & 0.26 & 0.26 & 0.19 \\
& L parietal & $0.65 \dagger$ & $0.61^{*}$ & 0.30 & $0.72 \ddagger$ & 0.15 \\
\hline \multirow{2}{*}{ * $p<0.025, \dagger p<0.01, \neq p<0.003}$. & & & & \\
L, left; MEG, magnetoencephalography; MRI, magnetic resonance imaging; $R$, right. & \\
\hline
\end{tabular}

Table 3 The relative importance of MEG and MRI variables in predicting cognitive function and adaptive behaviour

\begin{tabular}{|c|c|c|c|c|c|c|}
\hline & \multicolumn{2}{|l|}{ MMSE } & \multicolumn{2}{|l|}{ CAMCOG } & \multicolumn{2}{|l|}{ FAST } \\
\hline & Adj $R^{2}$ & $\mathrm{p}$ Value & Adj $R^{2}$ & p Value & Adj $R^{2}$ & $\mathrm{p}$ Value \\
\hline (MRI) R hippocampus & $0.695(2)$ & 0.006 & $0.684(1)$ & 0.001 & $0.660(1)$ & 0.003 \\
\hline (MEG) L temporal lobe & $0.819(1)$ & 0.001 & $0.854(2)$ & 0.001 & $0.766(2)$ & 0.018 \\
\hline
\end{tabular}

Adj $R^{2}$, adjusted $R^{2}$ value for each predictor variable; CAMCOG: Cambridge cognitive examination; FAST, functional assessment scale; L, left; MEG, magnetoencephalography; MMSE, mini-mental examination (Spanish version); MRI, magnetic resonance imaging; $R$, right.

$\mathrm{p}$ Value: test of the significance of the independent contribution of the first (1) and second variable (2) to enter the regression equation.

used, one representing the number of late activity sources in the left temporal lobe and the other those in the left parietal lobe. Temporal lobe activity sources included those localised in the mesial temporal regions. These sources, however, were not found in all subjects and, given the relatively small group size, were not amenable to separate parametric analyses.

Each variable in the left hemisphere reflected the sum of activity sources in two latency windows (400 to 500 and 600 to $700 \mathrm{~ms}$ ) which reliably discriminated between the two groups, as reported previously. ${ }^{15}$ Like the MRI data, the MEG data were normalised on the basis of the total number of activity sources in the entire brain for each participant. In all, 10 correlation coefficients were computed (between each of the two MEG variables and each of the five MRI variables) and evaluated using a Bonferroni corrected $\alpha$ level of 0.05/10 $=0.005$. In general, strong positive correlations were found between the two MEG variables and the relative hippocampal volume bilaterally (table 2). The only coefficient that was considered statistically significant, however, was that between left temporal lobe activity (including hippocampal activity) and the relative volume of the left hippocampus, indicating that the smaller the number of late activity sources in the left temporal lobe areas, the greater the atrophy in the mesial aspects of the left temporal lobe.

Owing to the inherent difficulty in determining the anatomical borders of the parietal lobe, inter-rater reliabilities among radiologists were not sufficient to warrant the use of the parietal lobe volume measures in the analyses. Interestingly, however, there was a significant positive correlation between degree of activity in the left parietal lobe and the relative volume of the left temporal lobe. Given that the bulk of parietal activity was noted near the temporo-parietal junction, this finding is not surprising, in view of the close links between the left temporal and parietal lobes in language and memory functions.

\section{Relations between volumetric MRI, MEG, and neuropsychological data}

In a series of stepwise linear regression analyses we examined the relative significance of MEG and MRI volumetric measures as predictors of performance on the two tests of cognitive function (MMSE and CAMCOG) and the scale of everyday adaptive behaviours (FAST). Four variables that were found to differentiate reliably between the two groups were used in these analyses. The design included two volumetric
MRI measures (left and right relative hippocampal volume) and the two MEG measures used above (relative degree of activity in the left temporal and parietal lobes).

These analyses indicated that only two variables make significant independent contributions to the variability of each of three behavioural measures: the degree of activity in the left temporal lobe and the relative volume of the right hippocampus. Combined, the two variables accounted for $77-85 \%$ of the variance in each of the three measures (table 3 ).

\section{DISCUSSION}

In agreement with previous reports, ${ }^{14}$ patients with Alzheimer's disease consistently showed greater atrophy in the mesial temporal areas in both hemispheres than in other areas. Further, our data show that the degree of left hippocampal atrophy correlates strongly with the magnitude of regional activation of left temporal areas during a short term memory task. The smaller the size of the left hippocampus-adjusted for total intracranial volume-the smaller the number of consecutive activity sources in left temporal areas. Notably this activity was found exclusively during the late stages of neural processing of the target stimuli-that is, between 400 and $700 \mathrm{~ms}$ after stimulus onset. ${ }^{15}$ In a similar manner, the magnitude of late activity in left parietal regions is a strong correlate of the relative volume of the ipsilateral temporal lobe. This finding corroborates previous reports of predominant left hemisphere metabolic dysfunction in Alzheimer's disease. ${ }^{1}$ Moreover, the real time functional mapping capability of MEG extends these findings to suggest that this dysfunction is related to neurophysiological operations that are part of the brain mechanism, which at least in part, supports memory function.

We can offer two alternative explanations for the relation between the structural integrity of the mesial temporal cortices and the functional status of temporal and parietal lobe regions. First, it is possible that degenerative changes in the left entorhinal cortex early in the course of the disease ${ }^{5}$ impair critical functional connections between the neocortex and the hippocampal formation. ${ }^{23}$ The existence of a direct functional link between the two parts of the temporal lobe is also supported by a strong association between temporo-parietal hypometabolism and hippocampal atrophy..$^{10}$ A disconnection between the hippocampal complex in the left hemisphere and ipsilateral neocortical areas could seriously impair the 
functional integrity of the neural circuit involved in memory function. ${ }^{24}$ At the behavioural level this may have a major effect on the circuit's efficiency in accessing stored information, thus impairing memory function. At the same time the hypothesised disconnection could lead to reduced neurophysiological activation in left temporal and parietal areas during the performance of memory tasks, as noted in the present study.

A second and complementary hypothesis derives from the notion that normal short term or working memory function depends upon the functional integrity of all the components of the brain circuit involving the mesial ${ }^{25}$ as well as the lateral temporal and parietal lobe areas. ${ }^{26}$ According to current theoretical models, ${ }^{27}$ working memory involves several component processes, including a phonological loop, visuo-spatial representations, central executive processes, and an episodic buffer. In our study we used a task that poses heavy demands on the phonological loop, ${ }^{15}$ a process intimately linked to the temporoparietal areas. If any of these regions is affected by degenerative processes-such as neuronal cell loss, neurofibrillary tangles, and senile plaques that are routinely found in these regions in Alzheimer's disease ${ }^{28}$ - the ability of the circuit to support memory related functions will be impaired.

Given the strong positive correlation between left hippocampal volume and degree of late activity in the ipsilateral temporal lobe, it is not surprising that the variable that makes a significant independent contribution to predicting individual scores on general cognitive/behavioural measures is atrophy in the right hippocampus. This finding is consistent with previous reports of an association between bilateral mesial temporal lobe hypometabolism and performance on cognitive scales like the MMSE. ${ }^{29}$

The fact that hippocampal atrophy improves the value of MEG derived regional activation measures in predicting individual scores on the cognitive (MMSE, CAMCOG) and functional (FAST) scales establishes a direct link between cognitive impairment and anatomical-functional measures, and highlights the potential clinical significance of both types of measure for the early diagnosis of Alzheimer's disease. It should be noted, however, that the widespread concurrent use of both imaging modes is unlikely in the near future, given the relatively small number of MEG centres worldwide.

This study supports the view that the lack of late brain magnetic activity in the left temporo-parietal region in patients with Alzheimer's disease may be related to the degree of atrophy in mesial temporal lobe structures. The results highlight the importance of assessing the complex brain pathology underlying Alzheimer's disease using multiple brain imaging modes. In addition, a conservative analytical approach was adopted in order to keep the likelihood of type I error at a minimum. This approach, which was thought necessary in view the large number of post hoc comparisons performed, was designed to keep the rate of false positive results under control. The sensitivity of MEG/MRI measures can be improved in future investigations by increasing sample size in order to enhance power and reduce the rate of type II error.

\section{ACKNOWLEDGEMENTS}

This study was supported in part by a grant from 4-D Neuroimaging. We would like to thank Thomas Murphy for technical assistance on MEG data acquisition.

\section{Authors' affiliations}

F Maestú, A Fernández, C Amo, S Fernandez, T Ortiz, Centro de Magnetoencefalografía Dr Pérez Modrego, Universidad Complutense Madrid, Spain

J Arrazola, Department of Neuroradiology, Hospital Universitario San Carlos, Madrid

P G Simos, A Papanicolaou, Department of Neurosurgery, Vivian L Smith Center for Neurologic Research, University of Texas-Houston Medical School, Houston, Texas, USA
Pedro Gil-Gregorio, Departamento de Geriatría, Hospital Universitario San Carlos, Madrid

Competing interests: none declared

\section{REFERENCES}

1 Thompson PM, Mega MS, Woods RP, et al. Cortical change in Alzheimer's disease detected with a disease-specific population-based brain atlas. Cereb Cortex 2001:11:1-16.

2 Sass KJ, Buchanan CP, Kraemer S, et al. Verbal memory impairment resulting from hippocampal neuron loss among epileptic patients with structural lesions. Neurology 1995;45:2154-8.

3 Kidron D, Black SE, Stanchev P, et al. Quantitative MR-volumetry in Alzheimer's disease: topographic markers and the effects of sex and education. Neurology 1997;49:1504-12.

4 Golebiowski M, Barcikowska M, Pfeffer A. Magnetic resonance imaging-based hippocampal volumetry in patients with dementia of the Alzheimer type. Dement Geriatr Cogn Disord 1999;10:284-8.

5 Killiany RJ, Gomez-lsla T, Moss M, et al. Use of structural magnetic resonance imaging to predict who will get Alzheimer's disease. Ann Neurol 2000;47:430-9

6 Laakso MP, Hallikainen M, Hanninen T, et al. Diagnosis of Alzheimer's disease: MRI of the hippocampus vs delayed recall. Neuropsychologia 2000;38:579-84

7 Petersen RC, Jack CR, Xu YC, et al. Memory and MRI-based hippocampal volumes in aging and AD. Neurology 2000;54:581-7.

8 Kohler S, Black SE, Sinden M, et al. Memory impairments associated with hippocampal versus parahippocampal-gyrus atrophy: an MR volumetry study in Alzheimer's disease. Neuropsychologia 1998;36:901-14.

9 Wahlund LO, Julin P, Johansson SE, et al. Visual rating and volumetry of the medial temporal lobe on magnetic resonance imaging in dementia: comparative study. J Neurol Neurosurg Psychiatry 2000;69:630-5.

10 Yamaguchi S, Meguro K, Itoh M, et al. Decreased cortical glucose metabolism correlates with hippocampal atrophy in Alzheimer's disease as shown by MRI and PET. J Neurol Neurosurg Psychiatry 1997;62:596-600

11 Johnson SC, Saykin AJ, Baxter LC, et al. The relationship between FMR activation and cerebral atrophy: comparison of normal aging and Alzheimer's disease. Neuroimage 2000;1 1:179-87

12 Pekonenn E, Jääskeläinen IP, Hietanen M. Impaired preconscious auditory processing and cognitive functions in Alzheimer's disease. Clin Neurophysiol 1999:110:1942-7.

13 Berendse HW, Verbunt JPA, Scheltens $P$, et al Magnetoencephalographic analyses of cortical activity in Alzheimer's disease: a pilot study. Clin Neurophysiol 2000;111:604-12.

14 Fernández A, Maestú $\mathrm{F}$, Amo $\mathrm{C}$, et al. Focal temporo-parietal slow activity in Alzheimer's disease revealed by magnetoencephalography. Biol Psychiatry 56:764-70

15 Maestú F, Fernández A, Simos PG, et al. Spatio-temporal patterns of brain magnetic activity during a memory task in Alzheimer's disease. Neuroreport 2001;12:3917-21.

16 Lobo A, Ezquerra V. El minimental cognoscitivo: un test sencillo y práctico para detectar alteraciones intelectivas en pacientes médicos. Actas Luso Esp Psiquiat Psicol Med 1979;3:189-202.

17 Sarvas J. Basic mathematical and electromagnetic concepts of the biomagnetic problem. Phys Med Biol 1987;32:11-22.

18 Simos PG, Breier Jl, Maggio WW, et al. Atypical temporal lobe language representation revealed by MEG and intraoperative stimulation mapping. Neuroreport 1999;10:139-42.

19 Simos PG, Papanicolaou AC, Breier Jl, et al. Localization of language-specific cortex using MEG and intraoperative stimulation mapping. J Neurosurg 1999;91:787-96.

20 Breier JI, Simos PG, Zouridakis G, et al. Lateralization of cerebral activation in verbal and non-verbal recognition memory tasks using magnetoencephalography. Brain Topogr 1999;12:89-97.

21 Papanicolaou AC, Simos PG, Breier Jl, et al. Magnetoencephalographic mapping of the language specific cortex. J Neurosurg 1999;90:85-93.

22 Whitwell JL, Crum WR, Watt HC, et al. Normalization of cerebral volumes by use of intracranial volume: implications for longitudinal quantitative MR imaging. Am J Neuroradiol 2001;22:1483-9.

23 Lavenex $\mathbf{P}$, Amaral DG Hippocampal-neocortical interaction: hierarchy of associativity. Hippocampus 2000;10:420-30

24 Mitchell TW, Mufson EJ, Scheneider JA, et al. Parahippocampal tau pathology in healthy aging, mild cognitive impairment, and early Alzheimer's disease. Ann Neurol 2002;51:182-9.

25 Collete $\mathbf{F}$, Van der Linden $M$. Brain imaging of the central executive component of working memory. Neurosci Biobehav Rev 2002; 26: 105-25

26 Lee I, Kesner RP. Differential contribution of NMDA receptors in hippocampal subregions to spatial working memory. Nat Neurosci 2002;5:162-8.

27 Baddeley AD. Is working memory still working? Am Psychol 2001;56:851-64

28 Asahina M, Yoshiyama Y, Hattori T. Expression of matrix metalloproteinase-9 and urinary-type plasminogen activator in Alzheimer's disease brain. Clin Neuropathol 2001;20:60-3.

29 Rodriguez G, Vitali $P$, Calvini $P$, et al. Hippocampal perfusion in mild Alzheimer's disease. Psychiatry Res 2000;100:65-74. 